\title{
Effect of Localized Laser Treatment on Fatigue Performance of Single-Crystal Silicon Microstructures
}

\author{
Mohamed E. Mitwally, Toshiyuki Tsuchiya, Osamu Tabata, and Sherif Sedky ${ }^{1}$ \\ Department of Microengineering, Kyoto University, Kyoto Daigaku-Katusra, Kyoto 615-8540, Japan \\ ${ }^{1}$ Provost Office, The American University in Cairo (AUC), New Cairo, Fifth Settlement, Egypt
}

(Received September 1, 2015; accepted December 18, 2015)

Keywords: laser treatment, single-crystal silicon, surface roughness, fatigue, fracture

Microscale single-crystal silicon (SCS) fatigue test structures having a notch at the midsection were subjected to a localized laser treatment with a $1 \mathrm{~J} / \mathrm{cm}^{2}$ laser energy for 5 pulses while tilted by $45^{\circ}$. As-fabricated and laser-treated samples oriented along the $\langle 110\rangle$ direction were subjected to fatigue test using the same loading $\left(1.7^{\circ}\right.$ rotation angle $)$ and environmental $\left(25^{\circ} \mathrm{C}\right.$ and $50 \%$ relative humidity) conditions to study the effect of laser treatment on fatigue performance. As-fabricated samples showed an average fatigue life of $5.6 \times 10^{8}$ cycles with the data being scattered within an order of magnitude. On the other hand, laser-treated samples showed a significant variation in fatigue life. Two samples showed an improved fatigue performance, one sample showed a comparable performance to as-fabricated samples, and two samples fractured instantly as the fatigue test started. Samples that fractured instantly showed the development of protrusions at the notch tip after laser treatment. These protrusions might have caused stress concentration that led to premature failure. Protrusions might indicate fluctuations in laser energy, which could be due to sample misplacement; hence, a more precise control of sample placement is needed for a consistent improvement in fatigue life after laser treatment.

\section{Introduction}

Single-crystal silicon (SCS) is the main building material in the field of micro-electromechanical systems (MEMS). SCS elements are subjected to mechanical loading in MEMS applications such as pressure sensors, micromirrors, and accelerometers. These elements include beams and diaphragms that are in a free-standing state in order to perform the required functions of sensing and actuation, and are continuously subject to cyclic stresses. Accordingly, MEMS elements are required to have long fatigue life. SCS microstructures however were observed to undergo fatigue failure under cyclic loading in various studies. ${ }^{(1-9)}$ To improve the mechanical performance of MEMS devices, it is crucial to improve the fatigue performance of the free-standing SCS elements involved.

Many factors, such as crystal orientation, fabrication process and environment, , $^{(1-3,9-11)}$ were found to affect the fatigue performance of SCS microstructures. One main parameter that causes poor fatigue performance and short life is sidewall roughness resulting from fabrication. ${ }^{(2,10)}$ The effect of surface roughness on the fatigue behaviour of SCS microstructures has seldom been

"Corresponding author: e-mail: mohamed.elwi@guc.edu.eg 
studied. Ikehara and Tsuchiya ${ }^{(10)}$ investigated the effect of sidewall roughness on various samples by varying the sidewall roughness, and they showed that fatigue life increased with decreasing sidewall roughness. It was also shown in another study that SCS samples with smooth sidewalls showed no fatigue damage up to $7.5 \mathrm{GPa}$ in an oxygen-free environment unlike those with rough sidewalls tested under the same environmental conditions. ${ }^{(11)}$

To improve the sidewall roughness of SCS microstructures, different techniques, such as wet etching, ${ }^{(12,13)}$ thermal oxidation, ${ }^{(14,15)}$ hydrogen annealing ${ }^{(16-19)}$ and laser annealing, ${ }^{(20-24)}$ were investigated in the literature. Laser annealing has an advantage over other techniques since the entire chip carrying the structures to be treated will not be subjected to soaking in chemical solutions or annealing at high temperatures up to $1100{ }^{\circ} \mathrm{C}$ as in the cases of thermal oxidation and hydrogen annealing. Previous research studies dealing with the laser irradiation of free-standing structures have only employed low energy densities for adjusting beam curvatures and solving stiction problems, and it was claimed that high energies would lead to significant temperature gradients and strains that lead to failure. ${ }^{(25-28)}$ To improve sidewall roughness, higher energies exceeding the melting threshold of silicon are required in order to have smoother sidewalls. Another challenge is to expose sidewalls to the laser beam efficiently without shadowing from neighbouring structures. In a previous study, we demonstrated that a localized treatment at high energies reaching up to $4 \mathrm{~J} /$ $\mathrm{cm}^{2}$ was possible using a mask and projection optics. ${ }^{(24)}$ Efficient sidewall exposure was achieved by tilting the chip carrying the test structures. We also demonstrated in other studies that SCS microbeams oriented along different orientations showed a significant improvement in tensile strength after the high-energy localized laser irradiation of sidewalls. ${ }^{(29)}$ The samples however had flat sidewalls with no notches unlike typical fatigue test structures. Notches or changes in cross sections need a precise control of laser treatment parameters (energy and number of pulses) in order to improve the surface roughness while preserving the surface profile. In a previous study on notched fatigue test structures, high laser energies improved the surface roughness but distorted the notch profile; thus, a compromise in laser energy is needed. ${ }^{(24)}$

Accordingly, it is the purpose of this study to investigate the effect of the sidewall roughness improvement of the SCS microstructure on fatigue life. The localized laser irradiation of sidewalls is the technique chosen to improve the sidewall roughness. An optimum choice of laser parameters was made to improve the surface roughness while preserving the notch radius. The fatigue lives of as-fabricated and laser-irradiated samples were compared to investigate the effect of the laser treatment of sidewalls.

\section{Materials and Methods}

Figure 1 shows the test structure that is used to evaluate the fatigue performance of SCS. The encircled test part having a notch at its center is oriented along the $<110>$ direction on a (100) SOI wafer.

This test structure has been used extensively for fatigue studies by Ikehara and Tsuchiya. ${ }^{(1,3)}$ The test part has a length of $30 \mu \mathrm{m}$, a width of $10 \mu \mathrm{m}$ and a thickness of $5 \mu \mathrm{m}$. The notch has a depth of $4 \mu \mathrm{m}$ and a radius of $0.5 \mu \mathrm{m}$. The corresponding stress concentration factor is $2.87 .{ }^{(30)}$ The structure has a large fan-shaped mass with a radius of $250 \mu \mathrm{m}$ having two sets of comb electrodes on both sides and a deflection scale. Electrodes are $2 \mu \mathrm{m}$ in width and $1.5 \mu \mathrm{m}$ in gap. One set of comb electrodes is used for actuation, while the other is used for sensing while the device is 


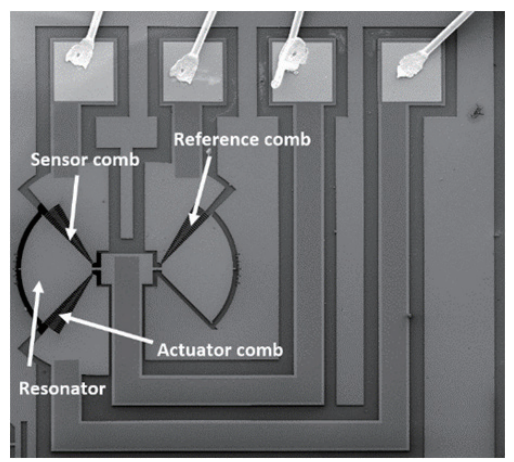

(a)

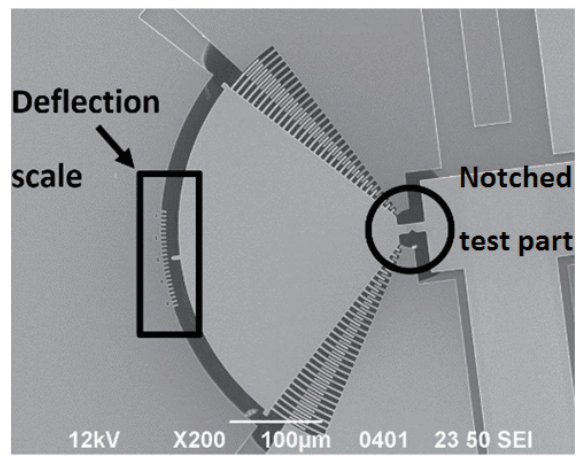

(b)

Fig. 1. Fatigue test device: (a) entire chip view showing different components and (b) zoom-up view of resonator showing notched test part and deflection scale.

tested under cyclic in-plane loading. The mass provides an adequate vibration amplitude during resonance. The design resonance frequency is $40 \mathrm{kHz}$. Differential sensing is used to detect the output by comparing the signal from the moving sensor comb with that from the identical reference comb. A deflection scale is used to measure the deflection angle during actuation. Details about structure fabrication can be found elsewhere. (5,6) $^{-}$

High-magnification images were taken at the sidewall as shown in Fig. 2. It seems that the sidewalls and notch exhibited a significant degree of scalloping due to the fabrication process.

Samples were irradiated using a Coherent LAEX-1000 KrF (248 nm) excimer laser source with a pulse duration of $30 \mathrm{~ns}$. A mask was used to have a nominal spot size of $100 \mu^{2}$ to cover only the notch area. More details about the laser treatment setup can be found elsewhere. ${ }^{(29)}$

Samples were attached to a sample holder, which was mounted on a two-axis stage for alignment. The pulse rate was kept constant at $1 \mathrm{~Hz}$ and the experiments were performed in air at $1 \mathrm{~atm}$ and room temperature. For the efficient exposure of sample sidewalls, the chips carrying the samples to be irradiated were tilted. Figure 3 shows a schematic of the treatment setup.

Only the side having the notch was irradiated since the crack is supposed to initiate at the notch. The optimized laser treatment conditions were $1 \mathrm{~J} / \mathrm{cm}^{2}$ for 5 pulses at a tilt angle of $45^{\circ}$ since they preserve the notch radius after treatment, which is crucial for a valid comparison between as-fabricated and laser-treated samples. A sample treated under such conditions is shown in Fig. 4. Higher energies lead to protrusions acting as locations of stress concentration at the notch tip, as shown in Fig. 5, for a sample irradiated at $1.4 \mathrm{~J} / \mathrm{cm}^{2}$ for 2 pulses at a tilt angle of $65^{\circ}$. Lower energies showed no significant improvement in roughness.

Owing to the significant effect of the environment on fatigue performance, the fatigue testing of as-fabricated and laser-treated samples was performed in a controlled environment oven at a temperature of $25{ }^{\circ} \mathrm{C}$ and a relative humidity of $50 \%$. First, a sweep test was performed over a range of frequencies to determine the exact resonance frequency of the sample. The actuation voltage was then varied to calibrate the relationship between the deflection angle of the resonator and the applied voltage by monitoring the deflection scale on the mass under an optical microscope. A deflection amplitude of $1.7^{\circ}$ was then used for both as-fabricated and laser-treated samples by 


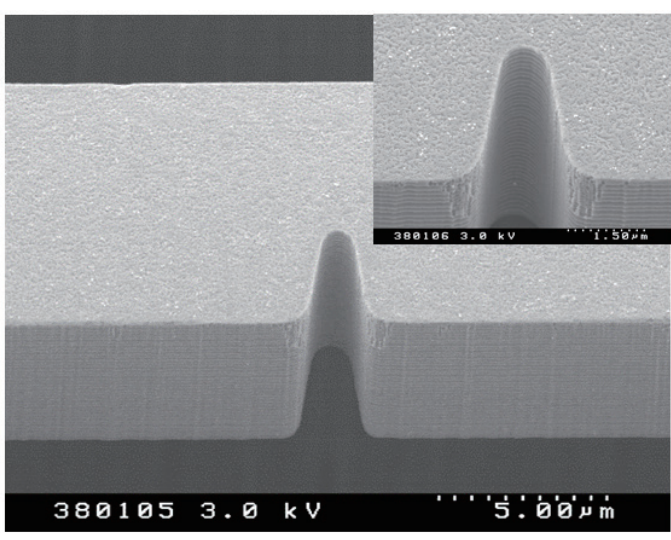

Fig. 2. Notch part in the middle of test part.

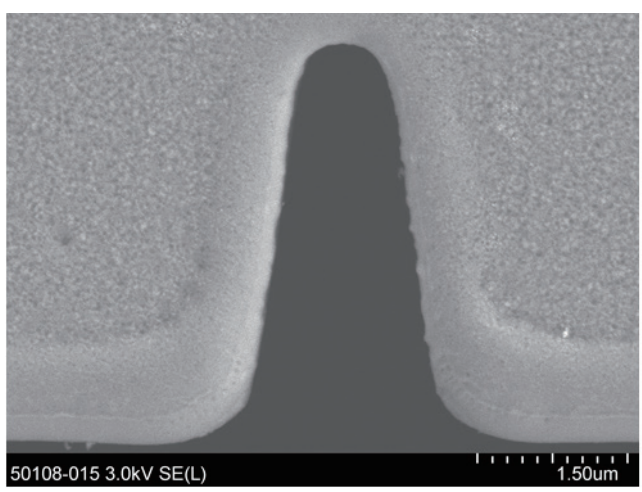

(a)

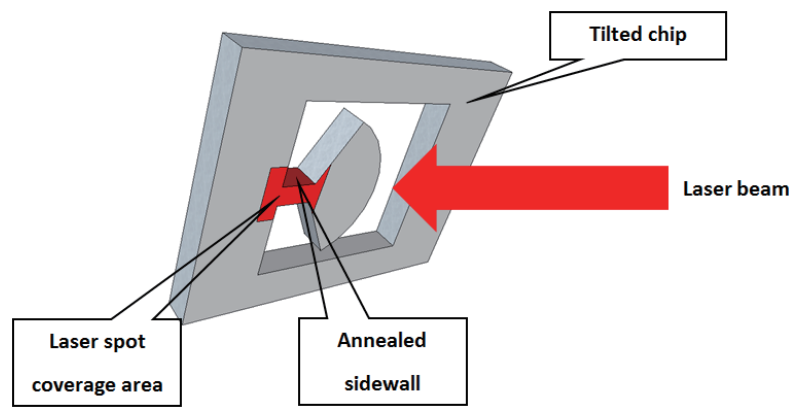

Fig. 3. (Color online) Schematic of treatment setup. The sample is tilted to irradiate the sidewall with the notch.

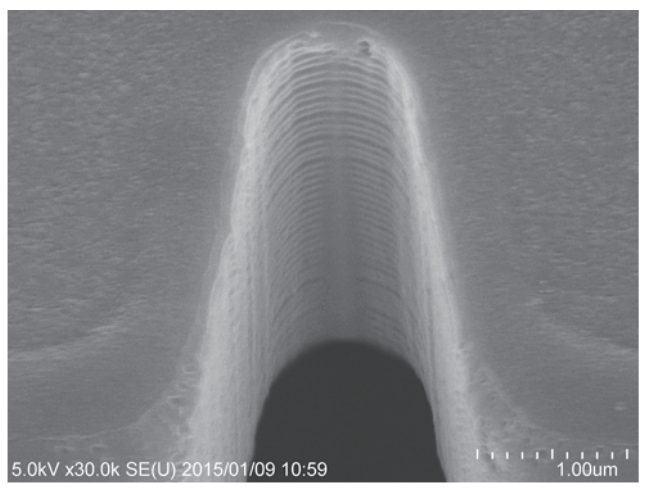

(b)

Fig. 4. Sample treated at $1 \mathrm{~J} / \mathrm{cm}^{2}$ for 5 pulses at a $45^{\circ}$ tilt: (a) top and (b) oblique views at $30^{\circ}$.

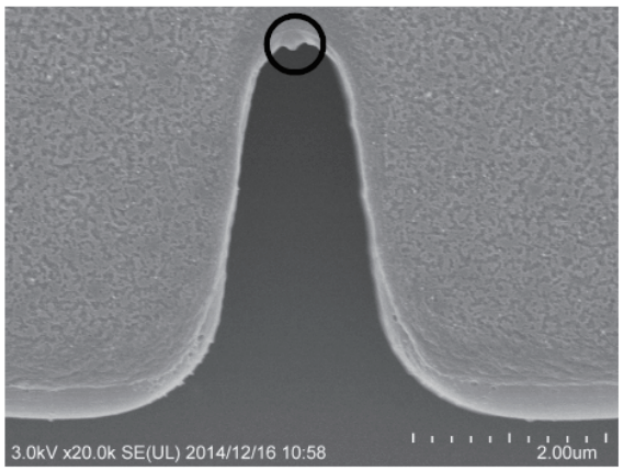

Fig. 5. Top view in notch area of a sample irradiated at $1.4 \mathrm{~J} / \mathrm{cm}^{2}$ for 2 pulses tilted at $65^{\circ}$ with the protrusion encircled.

applying a fixed corresponding actuation voltage. All samples were examined while resonating under the microscope to make sure no significant deviation in the deflection amplitude occurred. The output signal amplitude was recorded over time on a computer via an rms-dc converter to 
determine the maximum number of cycles after which no output is observed (fatigue life). More details about the fatigue testing circuit can be found elsewhere.(6)

The sidewall morphology and fracture surfaces of fatigue samples were evaluated from field emission scanning electron microscopy (FESEM) (Hitachi SU-8000 and S-4500) images. The fatigue life and fracture behaviour of seven as-fabricated samples were compared with those of five laser-treated samples.

\section{Results}

The fatigue lives of as-fabricated samples are shown in Table 1, while FESEM images for selected fractured samples are shown in Fig. 6. The number of cycles until fracture of seven asfabricated samples ranged from $8.3 \times 10^{7}$ to $1.3 \times 10^{9}$ with an average of $5.6 \times 10^{8}$.

Table 1

Fatigue lives of as-fabricated samples.

\begin{tabular}{cc}
\hline Sample ID & Fatigue life $\left(\times 10^{9}\right)$ \\
\hline 1 & 1.3 \\
2 & 1.1 \\
3 & 0.71 \\
4 & 0.45 \\
5 & 0.19 \\
6 & 0.13 \\
7 & 0.083 \\
\hline
\end{tabular}

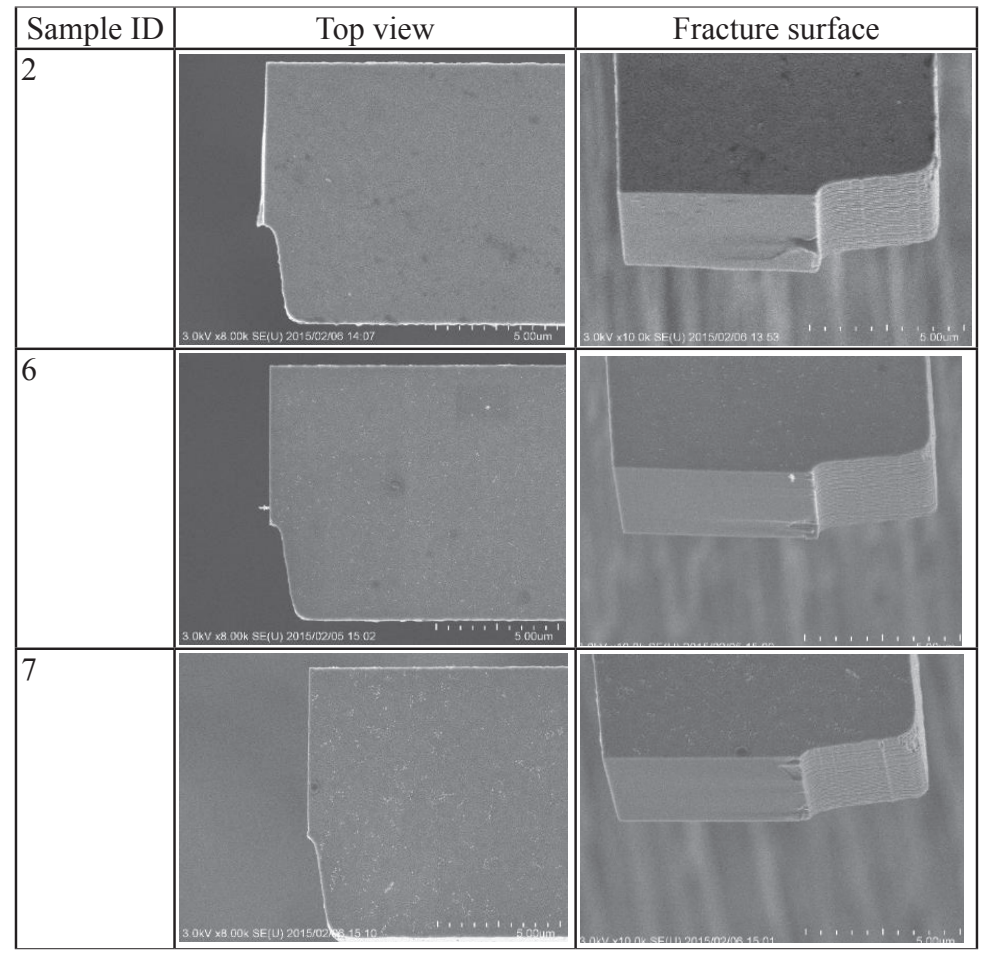

Fig. 6. Fracture surfaces of as-fabricated samples. 
The fatigue lives of laser-treated samples are shown in Table 2 with the corresponding FESEM images of selected fractured samples shown in Fig. 7. Laser-treated samples showed a wide variation in fatigue life. Two samples fractured instantaneously, one sample showed a fatigue life of 1.1 $\times 10^{7}$ cycles and two samples showed an improvement $\left(1.8 \times 10^{9}\right.$ cycles $)$.

The fracture behaviour of as-fabricated and laser-treated samples showed that the main cleavage plane was the (110) plane leaving smooth fracture surfaces as can be seen in Figs. 6 and 7.

Table 2

Fatigue lives of laser-treated samples.

\begin{tabular}{cc}
\hline Sample ID & Fatigue life $\left(\times 10^{9}\right)$ \\
\hline 1 & 1.8 \\
2 & 1.8 \\
3 & 0.011 \\
4 & Instant fracture \\
5 & Instant fracture \\
\hline
\end{tabular}

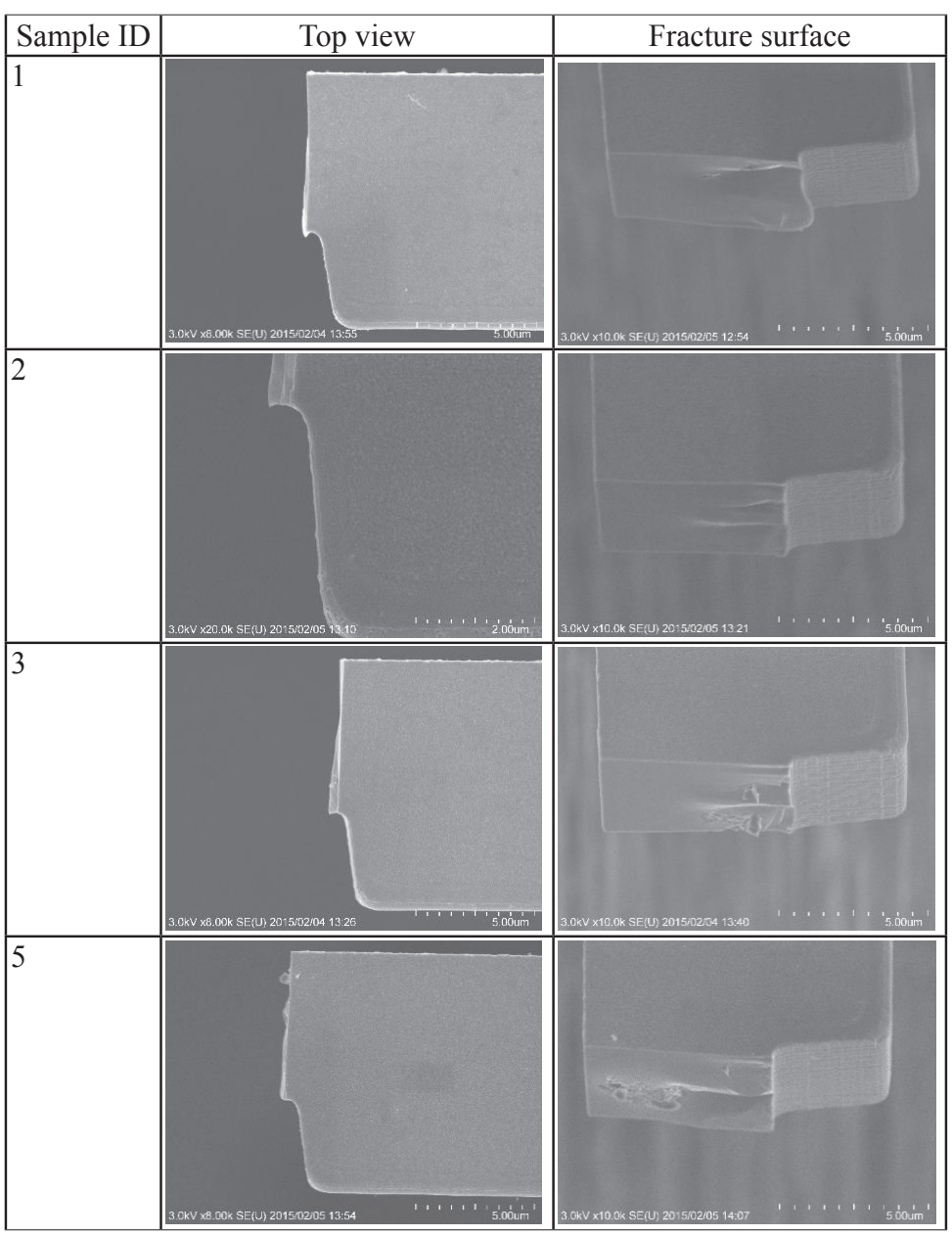

Fig. 7. Fracture surfaces of laser-treated samples. 


\section{Discussion}

For as-fabricated samples, results agree well with those of a previous study conducted by Ikehara and Tsuchiya, ${ }^{(3)}$ in terms of the fracture behaviour of similar samples. Also, the scatter in fatigue life found by Ikehara and Tsuchiya ${ }^{(3)}$ was within one order of magnitude, which is comparable to the scatter obtained in this study. Regarding fracture behaviour, from top views in Fig. 6, most samples had cracks initiating almost at the notch tip. This is attributed to the maximum first principal stress occurring at the notch tip as explained by Ikehara and Tsuchiya. ${ }^{(3)}$ Sample 7 with the shortest fatigue life fractured at an offset position from the notch tip, and we think that stress concentration at that location might have led to the shortest fatigue life.

As for laser-treated samples, looking at the top-view images in Fig. 7, the relationship between the fracture origin and the fatigue life was opposite to that for as-fabricated samples. In the lasertreated samples, the two samples that showed an improvement in fatigue life fractured at an offset position from the notch tip, whereas the three samples that showed a shorter fatigue life or an instant fracture fractured at the notch tip.

For the two samples with improved fatigue lives, the laser treatment was successful in improving the surface roughness at the notch tip and the crack initiation location shifted from the notch tip to an offset position. This proves the effectiveness of the localized laser treatment in improving the fatigue performance of SCS structures.

From the close-up image of sample 3 in Fig. 8, we see no effect of laser treatment in improving scallops in the notch area. We conclude that the sample was in an "as-fabricated" state in terms of roughness in the notch area and showed a fatigue life that was comparable to those of as-fabricated samples.

Samples 4 and 5 exhibited an instantaneous fracture. The top-view and oblique-view images taken in the notch area of sample 5 are shown in Figs. 9(a) and 9(b), respectively. In Fig. 9(a), a small protrusion encircled at the notch tip can be observed. However, the laser treatment was successful in improving the roughness at the notch tip as can be seen from Fig. 9(b). We suspect that the degraded fatigue life is due to the small protrusion at the notch tip as was observed in preliminary experiments where protrusions were observed at the notch tip of samples irradiated at a slightly higher energy $\left(1.4 \mathrm{~J} / \mathrm{cm}^{2}\right)$ and a higher angle $\left(65^{\circ}\right)$ shown in Fig. 5.

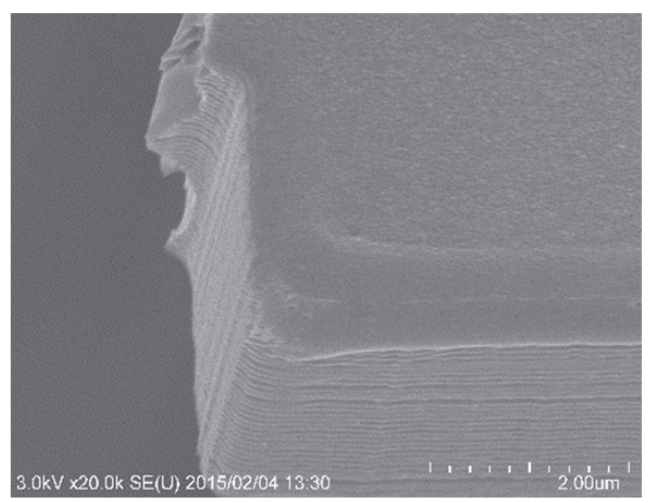

Fig. 8. Notch area of laser-treated sample 3. 


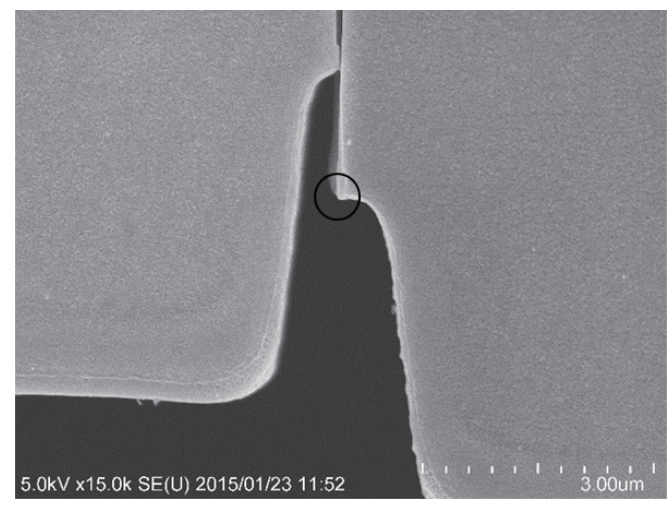

(a)

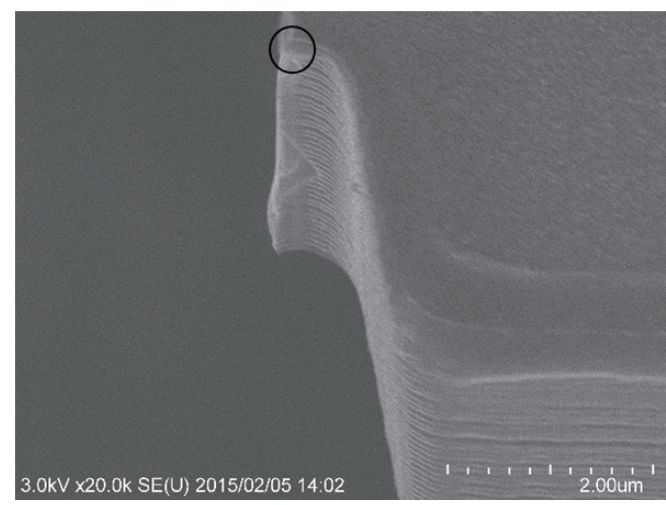

(b)

Fig. 9. Notch area of laser-treated sample 5: (a) top view just after fracture. The protrusion is encircled. (b) Oblique view after removal of mass side. The corresponding protrusion is also encircled.

Although the exact increase in stress amplitude due to the protrusion was not computed, however the failure mode is supposed to be due to stress concentration in tensile mode since a tensile load would lead to crack opening and accordingly failure.

The development of protrusions at the notch tip at the chosen laser energy $\left(1 \mathrm{~J} / \mathrm{cm}^{2}\right)$ of some samples can indicate a fluctuation in energy during irradiation. This could be due to a variation in sample orientation along the $45^{\circ}$ tilt plane or a variation in the position of the test structure to be irradiated within the laser spot, which might cause energy fluctuation. Accordingly, more precise control over sample positioning and placement is required to reduce the variation in fatigue life to have an improvement for all laser-treated samples.

\section{Conclusions}

A fatigue test of notched as-fabricated and laser-treated fatigue samples was conducted. A laser treatment at an energy that led to surface melting was successfully conducted owing to the localized irradiation in the notch area without fracture. The laser treatment led to an improvement in fatigue life in two of the five tested samples. The two samples showed fatigue lives that were an order of magnitude higher than the average fatigue life of as-fabricated samples and about $40 \%$ higher than the maximum fatigue life of as-fabricated samples. A large variation in the fatigue life of the five tested laser-treated samples was observed. Two laser-treated samples fractured instantly, which might be due to stress concentration observed at the notch tip. A better sample positioning could help reduce such variation.

\section{References}

1 T. Ikehara and T. Tsuchiya: Micro. Nano. Lett. 5 (2010) 49.

2 O. N. Pierron and C. L. Muhlstein: J. Microelectromech. Syst. 15 (2006) 111.

3 T. Ikehara and T. Tsuchiya: Proc. 2008 IEEE 21st Int. Conf. Micro Electro Mechanical Systems (IEEE, Tucson, 2008) p. 435.

4 H. Kahn, R. Ballarini, and A. H. Heuer: Curr. Opin. Solid State Mater. Sci. 8 (2004) 71. 
5 T. Ikehara and T. Tsuchiya: IEICE Electron. Express 4 (2007) 288.

6 T. Ikehara and T. Tsuchiya: J. Micromech. Microeng 18 (2008) 075004.

7 C. L. Muhlstein, S. B. Brown, and R. O. Ritchie: J. Microelectromech. Syst. 10 (2001) 593.

8 T. Ikehara and T. Tsuchiya: J. Microelectromech. Syst. 21 (2012) 830.

9 P.-O. Theillet and O. N. Pierron: Appl. Phys. Lett. 94 (2009) 181915.

10 T. Ikehara and T. Tsuchiya: IEEJ Trans. Sens. Micromachines 134 (2013) 32.

11 V. Hong, S. Yoneoka, M. W. Messana, A. B. Graham, J. C. Salvia, T. T. Branchflower, E. J. Ng, and T. W. Kenny: J. Microelectromech. Syst. 24 (2015) 351.

12 M. Shikida, T. Hasegawa, K. Hamaguchi, and K. Sato: Microsyst. Technol. 19 (2013) 547.

13 M. Shikida, Y. Niimi, T. Hasegawa, T. Sugino, S. Hamaoka, and K. Fukuzawa: Microsyst. Technol. 21 (2015) 661.

14 K. K. Lee, D. R. Lim, L. C. Kimerling, J. Shin, and F. Cerrina: Opt. Lett. 26 (2001) 1888.

15 J. Takahashi, T. Tsuchizawa, T. Watanabe, and S. Itabashi: J. Vac. Sci. Technol. B 22 (2004) 2522.

16 M. M. Lee and M. C. Wu: J. Microelectromech. Syst. 15 (2006) 338.

17 S. Kim, T. M. Roh, J. Kim, I. Y. Park, J. W. Lee, J. G. Koo, I. Bae, and K. I. Cho: J. Cryst. Growth 255 (2003) 123.

18 H. Kuribayashi, R. Hiruta, R. Shimizu, K. Sudoh, and H. Iwasaki: Jpn. J. Appl. Phys. 43 (2004) L468.

19 R. Hajika, S. Yoshida, W. Makishi, Y. Kanamori, S. Tanaka, and M. Esashi: Proc. 2013 IEEE 26th Int. Conf. Micro Electro Mechanical Systems (IEEE, Taipei, 2013) p. 425.

20 A. Bosseboeuf, J. Boulmer, and D. Débarre: Appl. Surf. Sci. 109 (1997) 473.

21 S. Sedky, R. T. Howe, and T. King: J. Microelectromech. Syst. 13 (2004) 669.

22 S. Hung, E. Liang, and C. Lin: J. Lightwave Technol. 27 (2009) 887.

23 E. Liang, S. Hung, Y. Hsieh, and C. Lin: J. Vac. Sci. Technol. B 26 (2008) 110.

24 M. E. Mitwally, T. Tsuchiya, O. Tabata, and S. Sedky: J. Surf. Eng. Mater. Adv. Technol. 5 (2014) 28.

25 J. W. Rogers and L. M. Phinney: J. Microelectromech. Syst. 10 (2001) 280.

26 J. W. Rogers and L. M. Phinney: Numer. Heat Tr. A-Appl. 45 (2004) 737.

27 X. R. Zhang and X. Xu: Appl. Phys. Lett. 86 (2005) 021114.

28 M. Dirscherl, G. Esser, and M. Schmidt: J. Laser Micro. Nanoeng. 1 (2006) 54.

29 M. E. Mitwally, T. Tsuchiya, O. Tabata, and S. Sedky: Microsyst. Technol. (2015) 1.

30 W. D. Pilkey and D. F. Pilkey: Peterson's Stress Concentration Factors (John Wiley and Sons Inc., New Jersey, 2008). 